\title{
Conduction tissue changes associated with enlarged membranous septum - a cause of sudden death?
}

\author{
W M I SMEETON, K R ANDERSON, S Y HO^, M J DAVIES, R H ANDERSON* \\ From the Department of Pathology, St George's Hospital Medical School; and Department of Paediatrics, \\ Cardiothoracic Institute, Brompton Hospital, London
}

SUMMARY We have studied four hearts in which enlargement of the ventricular membranous septum was associated with histological abnormalities of the atrioventricular conduction tissues. Two of the cases showed unusually formed penetrating and non-branching main atrioventricular bundles. These two cases plus one other also showed right-sided penetrating and main atrioventricular bundles. In addition these three cases all showed extensive fibre loss affecting the origin of the left bundle, which in one heart was further accompanied by fibre loss in the main bundle. The conduction tissue damage in the fourth case, though more extensive, was not as clearly related to the enlarged membranous septum since the heart in this case was also enlarged and dilated (congestive cardiomyopathy). Our findings, while obviously not conclusive, suggest that conduction changes in the presence of an enlarged membranous septum could be added to the list of causes of sudden cardiac death. They certainly indicate that when an enlarged membranous septum is found in cases of sudden death, histological study of the conduction system is desirable.

It is clear to physician and pathologist alike that a bewildering array of conditions are capable of causing sudden cardiac death. Usually, the pathologist is able to place the hearts from such cases into one of three categories $^{1}$ : firstly, ischaemic heart disease; secondly, conditions recognised macroscopically which are known to be associated with sudden cardiac death; and finally, hearts which are macroscopically normal. Cases in the last group, when, further, they show histologically normal contractile myocardium, pose a major problem and are undoubtedly more common than many pathologists readily care to admit.

There are, however, a number of hearts which perhaps form a twilight group between the second and third categories. There is a gross abnormality recognisable, but one, as yet, which has not been implicated as a cause of sudden death. Such a lesion is an unusually large membranous septum. The close relation between this structure and the atrioventricular conduction tissues is well recognised, so it is not unreasonable to suspect that lesions in this area may produce conduction problems.

We now have studied four hearts with macroscopic-

* Supported by the Joseph Levy Foundation together with the British Heart Foundation.

Received for publication 15 July 1981 ally recognisable abnormalities of the membranous septum; sudden death had occurred in three of the patients and documented atrioventricular block in the fourth. In this paper we describe our histological findings.

\section{Subjects and methods}

The method used for sectioning the atrioventricular conduction tissues has been previously described. ${ }^{2}$

\section{CASE A}

A 34-year-old woman was seen at an antenatal clinic at 14 weeks gestation one month before death. On examination her blood pressure was normal and there was no evidence of any other cardiac abnormality. A pregnancy 14 years previously had been uneventful. Seven years before she had an abortion at eight weeks gestation and three years before death a normal pregnancy complicated by a mild increase in blood pressure. The night before her death she was perfectly well and went to bed shortly after her husband. He awoke the next morning to discover her dead.

The post-mortem findings showed a gravid uterus, containing a male fetus of 18 weeks size which showed no congenital abnormalities. The patient's heart weighed $300 \mathrm{~g}$ with solitus atrial chambers and normal 
connections. There was no significant coronary vessel disease. The tricuspid valve was deficient medially in the region of the commissure between the septal and anterior leaflets. The membranous septum underlying this region was much enlarged, measuring $2 \times 1 \mathrm{~cm}$ (Fig. 1a). It is probable that the enlarged membranous septum in this heart was the result of tricuspid valve leaflet tissue closing a ventricular septal defect.

Histology of the atrioventricular conducting tissues showed a small but normal atrioventricular node with a normal transitional zone. The penetrating bundle and non-branching portion of the main atrioventricular bundle were right-sided structures. They were normal in size, though showing some fasciculation. The branching bundle, which was also a right-sided structure, ran within the lower margin of the enlarged membranous septum. This showed no fibre loss. The penetrating, non-branching and branching bundles were consequently long. The non-branching bundle was particularly elongated. The origin of the left bundle-branch showed complete interruption and fibre loss along its length. The distal left bundle-branch was never well-formed (Fig. lb). The fibre loss, however, was most convincing at the origin. The right bundle-branch was initially large but then rapidly became indistinguishable as a discrete fascicle.

\section{CASE B}

A 38-year-old woman presented with syncopal episodes 17 years before her death. An electrocardiogram at this time showed sinus rhythm with second degree heart block. She had previously had an atrial septal defect repaired. Five years after the first attack she suffered a further syncopal episode. A subsequent electrocardiogram recorded five years before death showed sinus bradycardia initiating ventricular fibrillation. She was therefore paced. She was also noted to have a long QT interval, and investigations at this time also showed a small left-to-right communication at ventricular level. She died suddenly.

The heart, which weighed $380 \mathrm{~g}$, was normal with the arch and aorta to the left. There had been a surgical repair of the atrial septal defect. The tricuspid valve was normally formed. The membranous septum beneath the valve was huge, being of the order of $2.5 \mathrm{~cm}$ in diameter. It completely closed the ventricular septum, but when viewed from the left

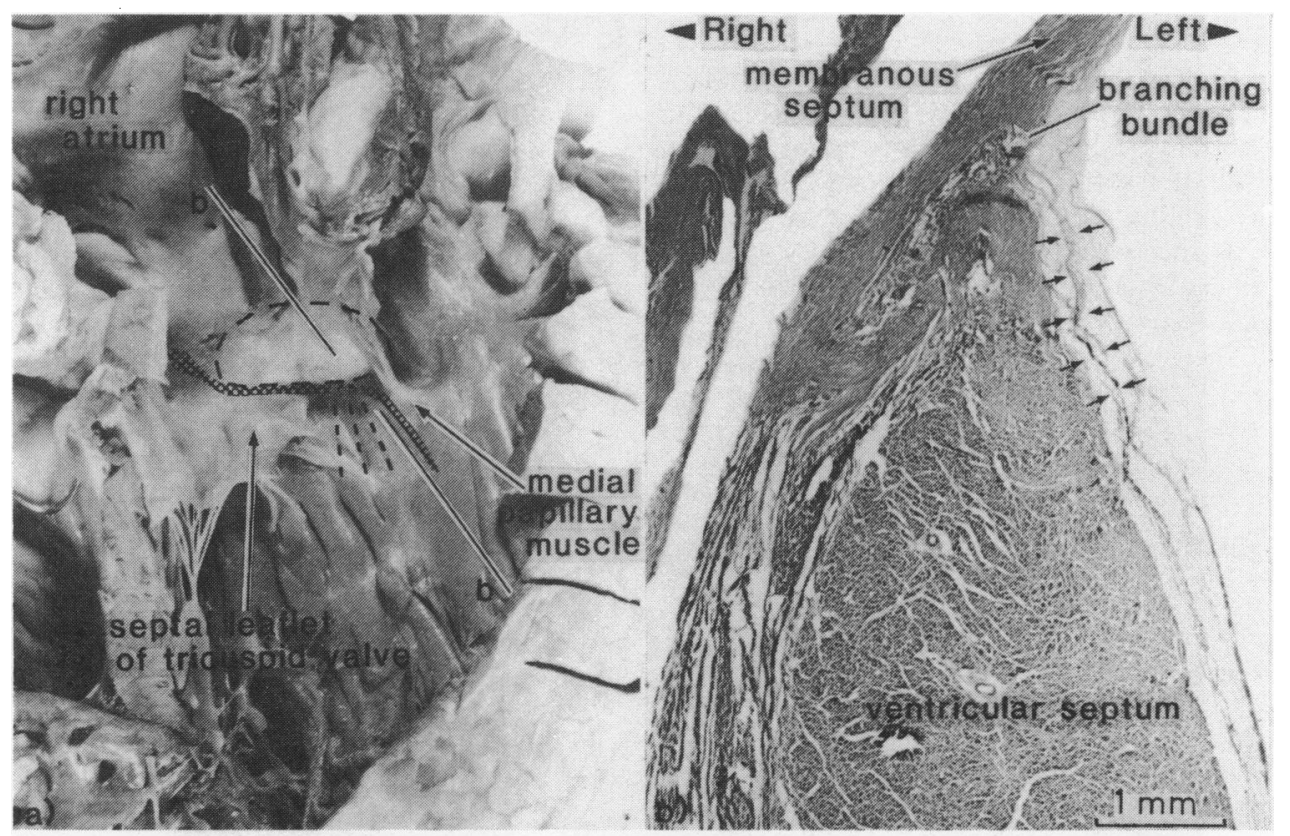

Fig. 1 (a) Photograph of the heart of case A viewed from the right side, showing the large membranous septum (enclosed within the dotted line) and associated deficiency of the tricuspid valve leaflet. The disposition of the conduction system has been superimposed on the photograph. Note the prominent non-branching component of the main atrioventricular bundle and the interrupted origin of the left bundle-branch. Plane b-b indicates level of sectioning in Fig. $1 b$. (b)Photomicrograph from the heart of case A of a section through the septal atrioventricular junction (plane b-b) showing a poorly formed left bundle-branch (arrows). (Masson's trichrome stain.) 
it was cicatrised, suggesting that initially there had been a small defect at this point (Fig. $2 a$ and $2 b$ ).

Histological examination of the atrioventricular conducting tissues showed a small but normal atrioventricular node. The transitional zones were sparse but probably adequate. The penetrating and nonbranching portion of the main bundle were most unusual. They formed a long plate-like structure overlapping the upper portion of the muscular septum on the right side (Fig. $3 \mathrm{~b}$ ). In the penetrating portion there was a ring of constriction though no complete interruption. As this structure progressed anteriorly it lost its plate-like formation and came to lie in a more usual position on the crest of the septum, but still positioned to the right. The branching bundle was fasciculated but showed no appreciable fibre loss. The origin of the left bundle-branch showed fibre loss and fibrous replacement over most of its origin (Fig. 3c). The left bundle-branch further down appeared normal. The right bundle was fasciculated but normal. The configuration of this unusual conduction system is shown diagrammatically in Fig. 3a. The sections also confirmed the presence of a small perimembranous ventricular septal defect. In addition branches of the nodal artery showed focal intimal thickening.
CASE C

A 50-year-old woman had suffered from palpitation since childhood. She had undergone an appendicectomy, a hernia repair, and one full term pregnancy without any: ill effect. The child had Down's syndrome with an atrioventricular septal defect. Over the last few years she had developed episodes of tachycardia. The electrocardiogram showed sinus tachycardia alternating with periods of atrial fibrillation, and evidence of left bundle-branch block. After treatment by beta-blockers, the rate came down. She later arrested, and, though initially resuscitated, developed left ventricular failure and died.

The heart, which weighed $380 \mathrm{~g}$, showed atrial situs solitus. The venous return was normal and there was atrioventricular concordance. There was no significant abnormality or narrowing of the coronary arteries. A large fossa ovalis had partially herniated into the left atrium, but the foramen ovale was closed. The most remarkable feature of the heart was the huge membranous septum measuring $3.0 \mathrm{~cm}$ in diameter (Fig. 4a) which was divided into a small atrioventricular and a larger interventricular component. A part of the septal leaflet of the tricuspid valve had become stuck onto the surface of the

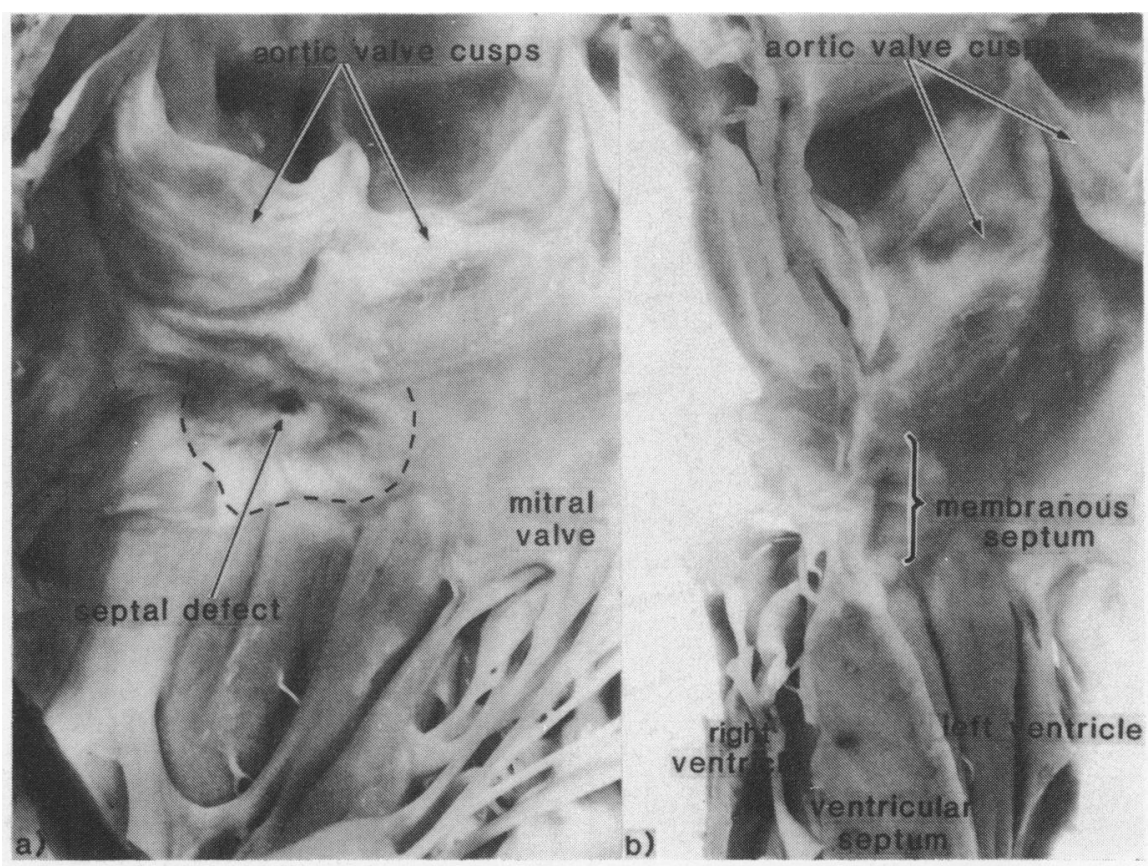

Fig. 2 (a) Photograph of the heart of case B viewed from the left side, showing the large membranous ventricular septum (enclosed within the dotted line) together with a small central membranous septal defect. (b) Photograph of the heart of case $B$ viewed from the anterior aspect to show the membranous and muscular ventricular septum. The heart has been cut in a plane at right angles to the septum just anterior to the small septal defect. 
Fig. 3 (a) Diagram illustrating the right side of the heart showing ihe configuration of the conduction system in the heart of case $B$. Note the unusual plate-like form of the penetrating bundle and non-branching main bundle and the interrupted origin of the left bundle-branch. Planes $b-b$ and $c-c$ indicate the levels of sectioning shown in Fig. $3 b$ and $3 c$, respectively. (b) Photomicrograph from the heart of case $B$ of a section through the septal atrioventricular junction (plane b-b in Fig. 3a). The arrows indicate the unusual plate-like configuration of nonbranching main atrioventricular bundle. (Masson's trichrome stain.) (c) Photomicrograph from the heart of case $B$ of a section through the septal atrioventricular junction (plane c-c in Fig. 3a) showing the sight-sided branching bundle with fibrous replacement of the origin of the left bundle-branch (arrows). (Masson's trichrome stain.)
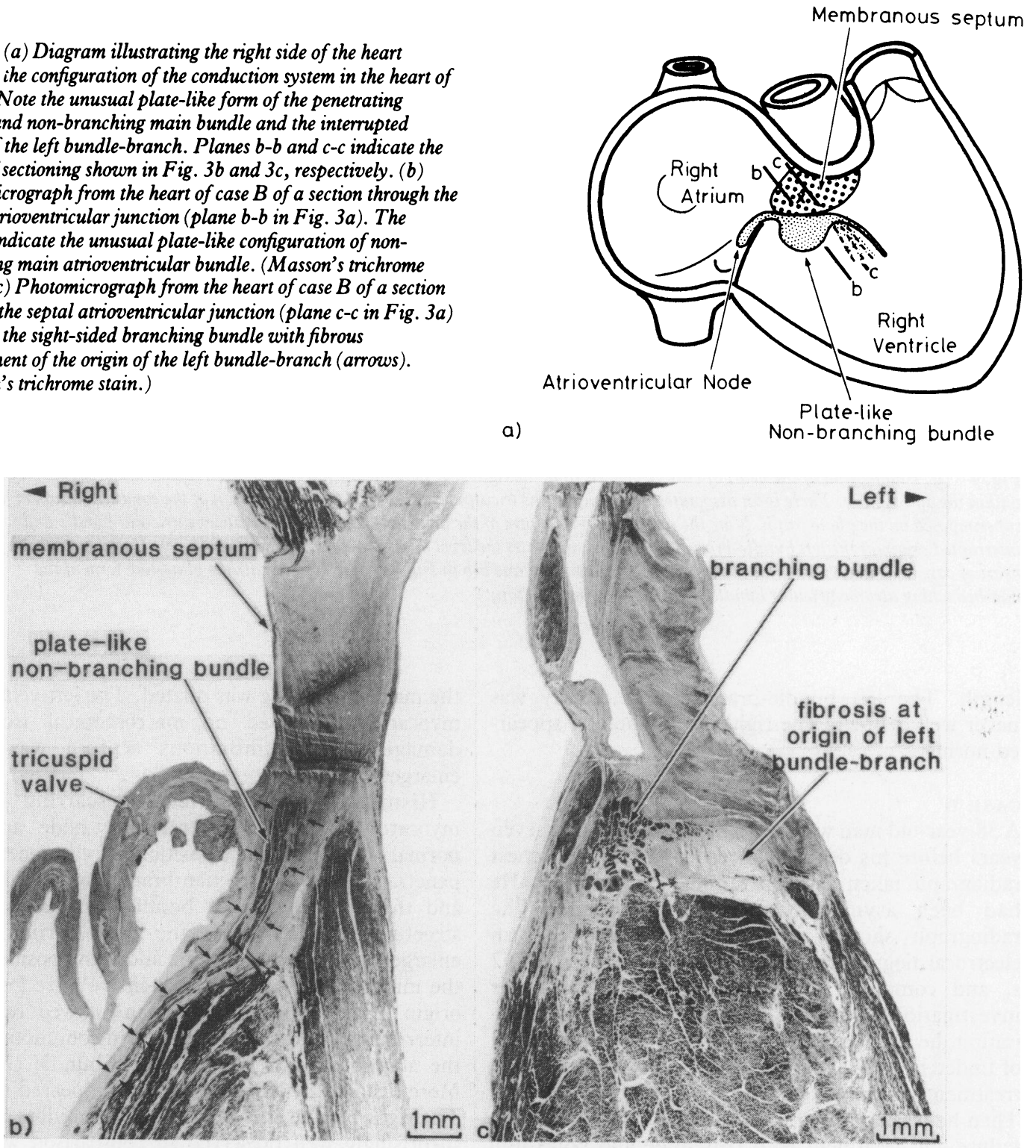

to a lesser extent than in case $B$, plate-like in form (Fig. 4b). The branching bundle, which ran within the lower portion of the enlarged membranous septum, was, however, nearer the midline. The main bundle (both branching and non-branching) and penetrating bundle formed an unusually long structure. The non-branching and branching main bundle showed extensive fibre loss (particularly the non-branching portion). The origin of the left bundlebranch also showed extensive fibre loss over its whole underlying enlarged membranous septum. On either side of this area, the valve leaflet again became freely mobile. The aortic valve was bicuspid but not stenotic. The origin of the right coronary artery was at the aortic bar, while the orifice of the left coronary artery, in contrast, was above the aortic bar.

Histology showed a normal atrioventricular node with normal transitional cell zones. The penetrating and non-branching portion of the main atrioventricular bundle were again right-sided and, though 


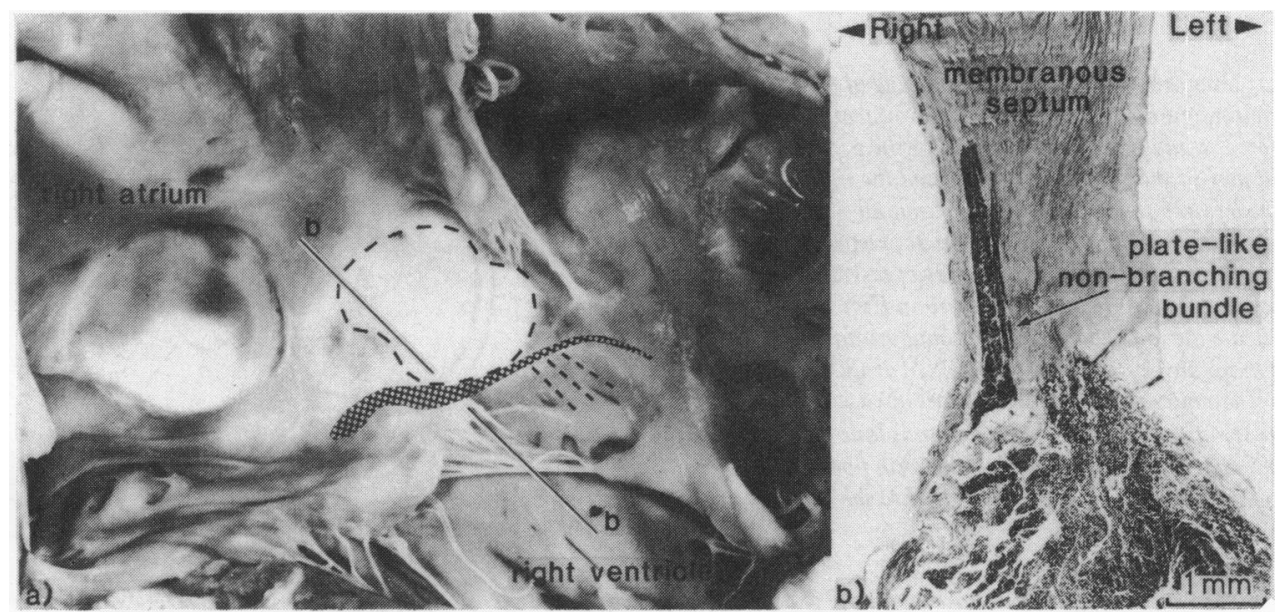

Fig. 4 (a) Photograph of the heart of case $C$ viewed from the right side showing the huge membranous ventricular septum (enclosed within the dotted line). There is an associated deficiency of the tricuspid valve leaflet. The disposition of the conduction system has been superimposed on the photograph. Note the rather plate-like form of the long non-branching main atrioventricular bundle and interrupted origin of the left bundle-branch. Plane b-b indicates the level of sectioning shown in Fig. $4 b$. (b) Photomicrograph from the heart of case $C$ through the septal atrioventricular junction (plane b-b in Fig. 4a) Arrows indicate the plate-like form of the non-branching atrioventricular bundle. (Masson's trichrome stain.)

length. The left bundle-branch more distally was never well defined. The right bundle-branch appeared normal.

\section{CASE D}

A 38-year-old man was first admitted to hospital seven years before his death as a result of a routine chest radiograph taken for superannuation purposes. He had been asymptomatic until this time. The radiograph showed cardiac enlargement and an electrocardiogram sinus rhythm, a PR interval of 0.2 $\mathrm{s}$, and complete left bundle-branch block. After investigations, which included cardiac catheterisation, he was diagnosed as having a cardiomyopathy of undetermined aetiology. He was discharged on no treatment and remained asymptomatic for five years. Then he was admitted to hospital in congestive heart failure and treatment with frusemide and potassium supplement was started. Though discharged improved, he was readmitted one month later with an exacerbation of cardiac failure and his treatment was therefore increased. The electrocardiogram showed sinus rhythm with $2: 1$ block and complete left bundle-branch block. He received a pacemaker implant and was again discharged only to be readmitted one week later with rapidly deteriorating cardiac failure which led to death.

The heart weighed $800 \mathrm{~g}$. All four chambers were dilated. The coronary arteries were involved by mild atherosclerosis. The heart valves were normal though the mitral valve ring was dilated. The left ventricular myocardium showed no macroscopical ischaemic damage. The membranous septum was much enlarged.

Histology showed extensive scarring of the myocardium. The atrioventricular node appeared normal with normal transitional cell zones. The penetrating bundle, the non-branching main bundle, and the branching main bundle formed elongated structures running within the lower portion of the enlarged membranous septum and being positioned in the mid-line. They showed extensive fibre loss. The origin of the left bundle-branch showed extensive interruption over its entire length in conjunction with the abnormality of the branching bundle (Fig. 5). More distally the left bundle never appeared normal. The right bundle also showed mild fibre loss but was surprisingly well preserved in comparison with the left bundle and branching bundle.

\section{Discussion}

When viewed from the left side, the normal membranous septum is an ovate thin fibrous membrane situated between the posterior and the right coronary leaflets of the aortic valve and the crest of the muscular ventricular septum. ${ }^{3}$ When viewed from the right side, it is crossed by the septal attachment of the tricuspid valve which divides it, in the majority of hearts, into atrioventricular and 


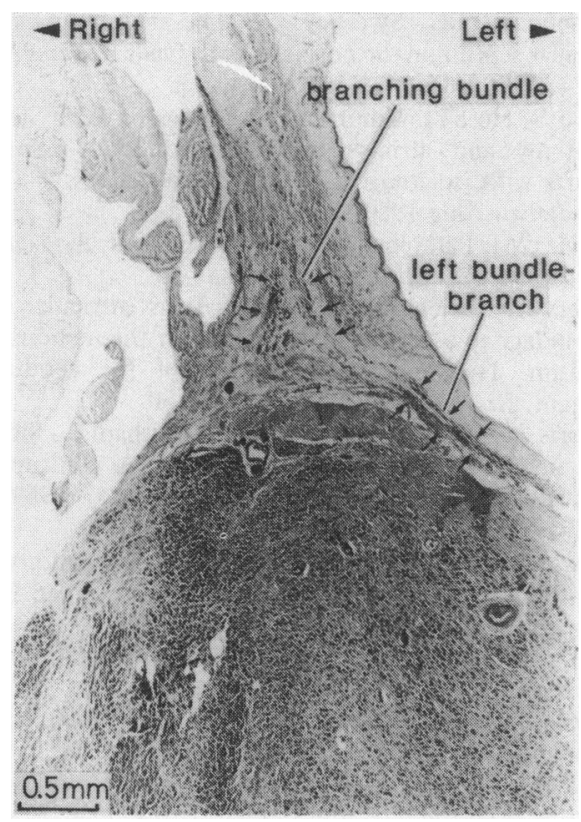

Fig. 5 Photomicrograph from the heart of case $D$ through the septal atrioventricular junction. Arrows indicate the branching bundle and origin of the left bundle-branch, both of which are represented only by a few sparse fibres. (Masson's trichrome stain.)

interventricular portions. The atrioventricular part lying above the tricuspid valve attachment relates the left ventricular outflow tract to the right atrium. The ventricular portion lying below the tricuspid annulus relates the left ventricular outflow to the right ventricle. Occasionally the annulus of the tricuspid valve is attached to the superior and posterior margin of the normally formed and situated membranous septum so that the atrial portion is absent. ${ }^{3}$

Because the membranous septum is related so closely to the atrioventricular conduction tissues, it should come as no surprise that malformation of the membranous septum may produce conduction tissue abnormalities. In the usual perimembranous septal defect, the main bundle passes down the posterior edge and usually branches on the left side of the inferior border. ${ }^{4}$ In atrioventricular septal defects the node is displaced posteriorly, elongating the main bundle. $^{5}$ The atrioventricular bundle also runs in the inferior margins of aneurysms of the membranous septum, and heart block is a recorded feature of this condition. ${ }^{6} 7$ Conduction abnormalities presumably arise after changes secondary to turbulent flow in the aneurysms.

In our four cases, there was unusual enlargement of the membranous septum with associated abnor- malities of the atrioventricular conduction tissues. Two of the cases showed an unusual plate-like formation of the penetrating bundle and nonbranching main bundle. This could only be developmental in origin, presumably occurring in conjunction with the forces which conspired to produce the enlarged membranous septum.

These two cases plus one other also showed right-sided penetrating and main atrioventricular bundles which, because of the large membranous septum, were unusually long. In these hearts there was a prominent non-branching component to the main bundle after it had penetrated the central fibrous body. A right-sided bundle can, in fact, be a variant of normal. Massing and James ${ }^{8}$ in a study of 32 normal hearts, noted that in five cases the His bundle coursed to the right of the interventricular septum. Further, they also noted that the left bundle in each of these cases began as a narrow stem which travelled to the left through the base of the membranous septum.

In addition, our cases all showed extensive fibre loss affecting the origin of the left bundle which in one heart was accompanied by fibre loss in the main bundle. Similar changes affecting the origin of the left bundle-branch can occur as an age-related phenomenon, possibly arising secondary to the pull of the myocardium and aorta on the fibrous skeleton in a high pressure chamber, ${ }^{9}$ so enlargement of the membranous septum may well accentuate such stresses producing an exaggerated or accelerated ageing process. A right-sided branching bundle with a narrow origin of the left bundle-branch could further enhance the likelihood of damage in this region. ${ }^{10}$

We have deliberately considered our fourth case separately because the changes in the conducting system are not as clearly related to the enlargement of the membranous septum. This case showed widespread fibre loss affecting the penetrating bundle, non-branching and branching main bundle, and left bundle. The right bundle was surprisingly wellpreserved, though showing mild fibre loss. The penetrating and non-branching and branching main bundle again formed a long structure, but situated this time in the mid-line in the lower portion of the membranous septum. This patient, however, had a large dilated heart (congestive cardiomyopathy) and conduction tissue damage is a documented feature of this condition, ${ }^{11}$ though severe involvement of the conduction system more usually affects only the more distal portions of both bundles.

Do the findings described in our first three cases represent a cause of sudden cardiac death? There are numerous published reports describing patients with abnormal conduction tissues who died suddenly. Lesions implicated range from such gross ones as mesotheliomas of the atrioventricular node to subtle 
histological changes ${ }^{12}$ which are accordingly more difficult to interpret. The interruption in continuity and fibre loss of the atrioventricular conduction tissues in our three cases are extensive and we suggest that they are capable of precipitating sudden death. Admittedly there are inherent problems in interpretation. Once having found a lesion there is a natural tendency to ascribe death to it. Correlation of morphology and function is difficult and positive findings do not necessarily imply abnormal function. A major problem is a knowledge of the range of normal and the extent of age-related changes. In addition, interpretation of conduction tissue abnormalities in the absence of electrocardiographic data (as in case A) has its dangers. On the other hand many argue that it is reasonable to draw a causal link when other possibilities are excluded. Studies of sudden cardiac death have undoubtedly shown a high incidence of conduction tissue abnormalities in hearts which are macroscopically normal. ${ }^{513}$

In summary, our findings while not conclusive, suggest that conduction tissue changes in the presence of an enlarged membranous septum should be added to the list of causes of sudden cardiac death. They certainly indicate that, when an enlarged membranous septum is found in cases of sudden death histological study of the conduction tissues is desirable.

\section{References}

1 Davies MJ. Pathological view of sudden cardiac death. Br Heart F 1981; 45: 88-96.

2 Smith A, Ho, SY, Anderson RH. Histological study of the cardiac conducting system as a routine procedure. Med Lab Sci 1977; 34: 223-9.
3 Rosenquist GC, Sweeney LJ. The membranous ventricular septum in the normal heart. Fohns Hopkins Med $\mathcal{F}$ 1974; 135: 9-16.

4 Milo S, Ho SY, Wilkinson JL, Anderson RH. Surgical anatomy and atrio-ventricular conduction tissues of hearts with isolated ventricular septal defects. $\mathcal{F}$ Thorac Cardiovasc Surg 1980; 79: 244-55.

5 Davies MJ. Pathology of chronic A-V block. Acta Cardiol (Brux) 1976; suppl 21: 19-30.

6 Thery C, Lekieffre J, Dupuis C. Atrioventricular block secondary to a congenital aneurysm of the membranous septum. Histological examination of the conducting system. Br Heart f 1975; 37: 1097-100.

7 Harris A, Davies M, Redwood D, Leatham A, Siddons $\mathrm{H}$. Aetiology of chronic heart block. A clinicopathological correlation in 65 cases. $\mathrm{Br}$ Heart $\mathcal{f}$ 1969; 31: 206-18.

8 Massing GK, James TN. Anatomical configuration of the His bundle and bundle-branches in the human heart. Circulation 1976; 53: 609-21.

9 Lev $M$. The pathology of atrioventricular block. Cardiovasc Clin 1972; 4: 159-86.

10 James TN, Schlant RC, Marshall TK. De Subitaneis Mortibus. XXIX. Randomly distributed focal myocardial lesions causing destruction in the His bundle or a narrow origin left bundle branch. Circulation 1978; 57: 816-23.

11 Davies MJ. Cardiomyopathy and chronic A-V block. In: Davies MJ, ed. Pathology of conducting tissue of the heart. London: Butterworths, 1971: 99-108.

12 James TN, Marilley RJ, Marriott HJL. De Subitaneis Mortibus. XI. Young girl with palpitations. Circulation 1975; 51: 743-8.

13 Gotoh K. A histopathological study on the conduction system of the so-called "Pokkuri disease" (sudden unexpected cardiac death of unknown origin in Japan). fpn Circ F 1976; 40: 753-68.

Professor R H Anderson, Department of Paediatrics, Cardiothoracic Institute, Brompton Hospital, Fulham Road, London SW3 6HP. 